\title{
Chatbot, as Educational and Inclusive Tool for People with Intellectual Disabilities
}

\author{
Montserrat Mateos-Sanchez ${ }^{1, *(1)}$, Amparo Casado Melo ${ }^{2}$ (1) , Laura Sánchez Blanco ${ }^{2}$ (1) \\ and Ana M. Fermoso García ${ }^{1}$ (D) \\ 1 Faculty of Computer Sciences, Pontifical University of Salamanca, 37002 Salamanca, Spain; \\ afermosoga@upsa.es \\ 2 Faculty of Education, Pontifical University of Salamanca, 37007 Salamanca, Spain; \\ acasadome@upsa.es (A.C.M.); lsanchezbl@upsa.es (L.S.B.) \\ * Correspondence: mmateossa@upsa.es
}

Citation: Mateos-Sanchez, M.; Melo, A.C.; Blanco, L.S.; García, A.M.F. Chatbot, as Educational and Inclusive Tool for People with Intellectual Disabilities. Sustainability 2022, 14, 1520. https://doi.org/10.3390/ su14031520

Academic Editors: José Antonio Marín-Marín, Santiago Alonso-García and Fernando José Sadio Ramos

Received: 2 December 2021 Accepted: 22 December 2021 Published: 28 January 2022

Publisher's Note: MDPI stays neutral with regard to jurisdictional claims in published maps and institutional affiliations.

Copyright: (c) 2021 by the authors. Licensee MDPI, Basel, Switzerland. This article is an open access article distributed under the terms and conditions of the Creative Commons Attribution (CC BY) license (https:/ / creativecommons.org/licenses/by/ $4.0 /)$.

\begin{abstract}
In the current health crisis due to COVID-19, people with intellectual disabilities have especially suffered. The development of their social abilities has been restricted, first with the lockdown and then with the current limitation of social life. They have lost some of these abilities or are having difficulty practicing them. CapacitaBOT, our use case, is a mobile application based on a chatbot, which allows people with intellectual disabilities to work and train their social skills. A chatbot is a software tool that allows to maintain a conversation in automatic way between the user and the machine, the mobile application. CapacitaBOT can be considered by its features, an educational ICT tool that introduces innovation, inclusion and quality in order to be integrated into education for people with intellectual disabilities. The tool trains these people for real-life situations and can also be considered a resource that allows the application of active methodologies since it makes easy the learning of social skills. In addition, all the contributions of the tool are aligned with the objectives of sustainable development because it is a tool that facilities the accessibility of people with disabilities, who more than ever have been affected by social isolation caused by the COVID-19 crisis.
\end{abstract}

Keywords: chatbot; mobile application; social abilities; inclusive education; intellectual disabilities; educational innovation; COVID-19

\section{Introduction}

As a result of the current health crisis, lockdown and subsequent de-escalation, people with disabilities have seen their social relations restricted, causing them to lose previously acquired social skills or to have more difficulties in putting them to use again.

During this period, people with disabilities who have had digital tools, mobile phones with applications, tablets or computers have been more connected than people who did not have these resources. For this reason, a technological tool has been developed based on the conversational bot that facilitates universal accessibility and allows the development of the social skills of people with intellectual disabilities, so that, they can lead a full life as normal as possible under the principle of inclusion.

People with intellectual disabilities meet communication, accessibility, cognitive and sensory barriers that complicate their access and participation in community services, accentuated by the current situation where contacts and opportunities to implement social skills in contexts other than the family are limited. Historically, people with intellectual disabilities faced barriers that complicated their access to participation in community services because of their difficulties in developing social skills. With regulatory changes and awareness campaigns, a more committed, inclusive and innovative society is being built, although there are still progress to be made, which can be strengthened with new methodologies and the use of innovative resources that improve the daily life of people with disabilities. 
This paper presents a solution based on conversational bots, as an educational and inclusive resource for people with intellectual disabilities, with the aim of continuing to provide solutions to advance in helping this group.

In this section, we will first define what is meant by intellectual disabilities and how technology can contribute to improve their social skills. Next, we will discuss how conversational bots, as a concrete technological solution to help people with intellectual disabilities, can contribute to this improvement, as well as their capacity for innovation in the field. Finally, it concludes with the objectives of the work.

\subsection{Intellectual Disability and The Need for Technological Solutions to Improve Their Social Skills}

The American Association on Intellectual and Developmental Disabilities notes: "Intellectual disability is characterized by significant limitations in both intellectual functioning and adaptive behaviour as manifested in adaptive, conceptual and practical skills. This disability originates before the age of $18^{\prime \prime}$ [1]. Intellectual functioning refers to the ability to think, to learn, to remember, to be aware of one's surroundings, to solve problems, etc. In addition, the World Health Organization (WHO) states that intellectual disability: "Intellectual disability is a disorder defined by the incomplete or arrested mental development of the person by having impaired the specific functions of each stage, which contributes to a low level of intelligence and affects cognitive, language, motor and socialization functions; therefore, adaptation to the environment is affected". The WHO also notes that "in order to determine the degree of development of intellectual disability, all information must be taken into account, including, of course, the clinical diagnosis, the subject's adaptation to the environment and psychometric findings" [2].

It is therefore necessary to provide support resources for the development of skills, the improvement of cognitive abilities and quality in education.

In Europe, there is great interest in removing new barriers for people with disabilities in the information society. This is only possible if ICTs are developed based on the principles of accessibility and "design for all". Authors such as Heredero and Carralero [3] argue that ICT are important tools for motivating cognitive aspects and essential in the case of people with disabilities. According to Cabero, Córdoba and Fernández [4], ICTs have a recognised capacity to promote social and educational integration:

"It is necessary to take into account that in order to make the most of the potential of technologies the school must use new ways of teaching and know what role ICT play in the education of people with special abilities, what are their possibilities of access and in the possibility of being part of the "information society" as another citizen" (p. 490).

Similarly, other organisations such as Save the Children [5] and UNESCO [6] have highlighted the need to provide people with disabilities with technological resources that favour inclusion and educational innovation. In this sense, the conversational bot offers a concrete response by reducing inequalities and ensuring the rights of persons with disabilities, promoting equal opportunities, educational inclusion and universal accessibility (SDGs 4, 5, 8 and 11).

As noted by the UN [7] in the Convention on the Rights of Persons with Disabilities, accessibility includes:

"[ ... ] appropriate measures to ensure accessibility for persons with disabilities, on an equal basis with others, to the physical environment, to transportation, to information and communications, including information and communications technologies and systems, and to other facilities and services open or provided to the public, both in urban and rural areas". (art. 9)

The main objectives of the Convention were to promote and guarantee, on an equal basis, the fundamental rights and freedoms of all persons with disabilities, and to try to overcome the barriers that persons with disabilities encounter, when interacting, that prevent them from full social participation.

Spain joined in the achievement of these objectives through the Instrument of Ratification of the Convention on the Rights of Persons with Disabilities, made in New York on 
13 December 2006, which subsequently came into force in our country on 3 May 2008 [8]. Consequently, accessible means must be promoted to guarantee equality for all people.

Learning social skills depends on maturation and learning experiences. People with an intellectual disability can acquire social skills in the same way as those who do not have them, although individual characteristics must be taken into account when planning the teaching-learning process.

For all of the above reasons, having technological tools available to help people with disabilities in the teaching-learning process is a great benefit. Some of these technological tools, which facilitate the tasks of people with learning difficulties, are: Hermes mobile Hermes Mobile-ASPACE (2014), which is an application for the communication of people with speech and literacy difficulties, the application is customizable, but is not able to interact intelligently with the user; or EmoPlay Emoplay is a digital tool aimed at people with autism, which allows to train the recognition of various emotions through facial expressions, making use of image or pattern recognition algorithms. Many others can be found in [9], however, according to the author, there is still much to be done and there is a lack of technological solutions to help people with intellectual disabilities in this learning process in the field of social relations. Therefore, this paper presents a new tool based on a conversational bot, which will help people with disabilities to develop and improve their social skills, facilitating the learning process for them.

\subsection{Conversational Bot as a Technological Solution for the Improvement of Social Skills in People with Intellectual Disabilities}

The concept of a bot is not new and already in 1997, this term was defined as an autonomous software program, endowed with personality and generally providing a service. Some authors [10-13] have explained them differently depending on the context, but all these definitions can be summarized as software or computer program made up of sets of rules that, working autonomously, automate specific tasks or processes and imitate human behaviour. Bots have been used intensively for certain tasks such as tracking information on the web, simulating traffic on the internet, automated editing of websites, assigning automated responses on websites or communicating by simulating a conversation with a human. This last use of conversational bots is the one of interest in this work.

A conversational bot [14] is software that simulates a conversation with a person by providing automatic responses to user input. In their early days, in the 1980s and 1990s, the first conversational bots, such as Eliza [15], A.L.I.C.E [16] were able to carry on a conversation by means of pre-prepared responses. Today, a distinction is made between bots that follow basic rules, similar to the first bots, and are therefore not very intelligent, and bots that are based on artificial intelligence. In unintelligent bots, the dialogue, i.e., the questions, as well as the possible answers, are defined, and they generally only understand a predetermined set of concepts related to their function, are not very efficient and provide a poor user experience. However, in intelligent bots, they try to mimic human language in all its complexity, making use of artificial intelligence systems with natural language processing engines and machine learning, in which the bot is trained with the help of human interaction. The latter, which use a combination of artificial intelligence techniques and real-time training or learning by a human, have the capacity to provide a more efficient service and a better user experience.

Currently, conversational services are widespread in contexts such as business and financial services [17-19], tourism services [20,21], communication services [12], messaging services or social networks [22], in psychology or medicine [23-25] or in education [26,27] among others. Some of them are closed. However, in the field of functional diversity such as Tess [28] a chatbot for mental health that pretends to be a therapist, exists, but does not work on social skills; as well as Chatbot COVID-19 from Semantics bots [29], which is very useful for people with visual impairment as it provides information about COVID-19, but does not train the user in the development of social skills. O Woebot [30] is a chatbot to provide cognitive behavioural therapy. However, no work has been found in which a 
conversational bot and the platforms that support them are used in the context of the scope of this research, so futher development of technological solutions is needed and, also for this reason, the use of these platforms in these contexts is considered to be innovative.

From all the works reviewed, it can be stated that a conversational bot is a software programmed to hold conversations with a person automatically and autonomously; the most advanced ones make use of artificial intelligence to learn by themselves in order to hold more natural, complex and appropriate conversations. These potentialities allow the development of projects and tools with which it is possible to interact with machines in a similar way to how people do, with natural language, in a precise manner, and they also have the capacity for training and learning. In this way, in this paper is presented this innovative solution that makes use of a conversational bot as a proposed ICT tool to educate people with intellectual disabilities to improve their social skills. With the tool, users could interact as if they were interacting with a human, and due to the automatic learning techniques, it will adapt to each user and their level of learning, as the tool will learn and adapt to their needs. Based on the observation protocols, conversation logics adapted to these users have been defined, but this logic will evolve with the use of the tool. As it is implemented on a mobile device, users will be able to practice with it at any time and in any place.

In this sense, the developed tool helps in learning processes, constitutes a didactic medium that favors the inclusion of people with intellectual disabilities by overcoming communication barriers, and also improving the quality of education with a teaching model focused on competency-based knowledge and the use of active methodologies that empower the subject, preparing them for real-life situations and thus achieving full participation. Furthermore, the use of accessible technologies for people with intellectual disabilities is developed in line with the Sustainable Development Goals (SDGs) established by the United Nations [31] in the 2030 Agenda

With this work, innovation in the educational process is achieved, favoring an inclusive and quality education, at the same time aligned with active methodologies by preparing the user for real life situations. Furthermore, facilitating accessibility to education through the use of new technologies is also in line with the sustainable development goals themselves. Finally, the proposal is particularly useful in the current situation of health crisis caused by COVID-19, where people with disabilities are particularly suffering from social isolation and, thanks to this tool, users can continue to work on their social skills.

For all of the above, the design and implementation of this intelligent tool can become a great ally of people with intellectual disabilities, because, with a conversational chat, integrated into a mobile application, the aim is to improve social skills and, therefore, greater social inclusion of people who train with it.

\subsection{Objectives of the Work}

In line with the above, the objectives pursued by this work are as follows:

- To Identify the needs of people with intellectual disabilities in order to provide them with a technological medium to help them communicate.

- To favour the educational and social inclusion of people with diverse functional abilities through the training and acquisition of (verbal) communication skills.

- To develop an intelligent conversational tool to train and acquire communication and interaction skills for people with intellectual disabilities.

- To improve the quality of life of people with intellectual disabilities by involving them.

The following sections first indicate the methodology used for the development of the project, followed by the results obtained, and finally a discussion of these results and the conclusions and final contributions of the work.

\section{Materials and Methods}

Twenty-five adults with intellectual disabilities, aged between 18 and 55, who are users of the Insolamis Association (http:/ /www.insolamis.org/ (accessed on 1 December 2021)), 
where they carry out daily activities for their educational and social inclusion, participated in this research. The meeting was coordinated by the technical support staff of the aforementioned organisation.

The main problem, which has started the research, is to discover which are the difficulties that limit the development of social skills to improve communication skills and thus facilitate the educational and occupational inclusion of people with intellectual disabilities. For its identification, being a qualitative study, social research techniques were used, such as semi-structured interviews and non-experimental observation.

For its implementation, 12 weekly sessions of 50 min were organised from October to December 2019 in the Insolamis Association, which is located in the city of Salamanca.

Once the data had been collected and the needs of Insolamis users had been identified, the work team led by the authors of this study, with knowledge in Computer Science and Pedagogy, proceeded to select the knowledge bank and the technical requirements demanded for the design and implementation of the chatbot, using a mobile application as a support. To this end, an analysis of development platforms was carried out beforehand and it was discovered that the best alternative is IBM's Watson.

The implementation of the chatbot dialogue is the fundamental task for the development of the tool, which offers log entries in voice or text format, allowing the most appropriate response to be given at any given moment. The result of this phase led to a first chatbot prototype.

In January 2020, a second training phase followed, in which training and tests were carried out with real users to check its functionality in the development of communication skills. The feedback allowed for improvement until the final product was obtained with the approval of the users, who became evaluators of the tool.

The exploitation and dissemination of the tool was not completed due to the lockdown (March 2020), but this fact increased, to a great extent, the need to provide technological resources to people with disabilities, because they are configured as didactic means, which favour the development of communicative and social skills.

In the following, the stages of development are presented in more detail and adjusted to the achievement of the tool:

\subsection{Situation Analysis by Collecting Data for the Identification of Social Needs of People with Disabilities}

Insolamis is a non-profit organisation that was set up in 1992 on the initiative of some families of people with intellectual disabilities. The main aim is the integral training to achieve their social and labour integration and the improvement of their quality of life. The working methodology is based on the "paradigm of Supports, Quality of Life model, self-determination and orientation towards the person". To this end, it has a Day Centre, where users receive comprehensive care during the day period, the main lines of action being health and safety, labour insertion, inclusion in the community and personal and social development. The Special Employment Centre (CEE) is another resource of the organisation, as users participate in market operations to try to facilitate gainful employment. Through the support units, Insolamis provides "personal and social adjustment services to working people with disabilities", taking into account their personal circumstances and the provisions of Royal Legislative Decree 1/29 November 2013. (BOE, 3-12-2013).

For the Promotion of Personal Autonomy (SEPAP) also has a service for adults with intellectual disabilities who have a recognised situation of dependency. When the intervention is carried out, the association offers four modalities: "habilitation and occupational therapy; cognitive stimulation; promotion, maintenance and recovery of functional autonomy; and psychosocial habilitation". Likewise, it has an Independent Living Service to promote social participation and it is made up of the personal assistant, supervised housing, and conciliation and family respite programmes.

The personal assistant service provides help in carrying out daily tasks, especially for people in a situation of dependency. The supervised housing service makes personal 
autonomy and independent living possible, although it is reinforced with support resources; conciliation and family respite allows family members to have their moments of rest, as they have the necessary support.

Insolamis also has a Family Care Service (SAF), which ensures the wellbeing of each of its members; a leisure and free time service to promote healthy habits and to enable them to interact with others in normalised environments; and a volunteer service and training practices, where people from different fields collaborate: Psychology, Pedagogy, Computer Science, among others.

The entity is known because several activities of social inclusion have been carried out with the support staff and with the users with intellectual disabilities. For this reason, Insolamis has been selected to carry out this research.

The entity is the main source of information, through the interview to professionals who provide their services in these associations, the training programmes have been known in detail, which are very useful data to follow a progression in the development of the capacities of the users with intellectual disabilities who voluntarily participate in the study.

Before each interview, from the research group, a work plan is designed, which is agreed with the association itself, with the points to be dealt with, as well as with the selection of people to be interviewed by the association, and always with their authorisation. The research group also schedules the observation protocols to collect the interviewees' answers. In this way, the team is able to specify the requirements of the tool, and to contrast its own conclusions with new interviews to confirm the correct understanding of the information gathered.

After this period of frequent interviews, the basic functionalities and features that the intelligent chatbot to be developed should have obtained.

\subsection{Analyse and Selection of the Technological Platform}

Almost parallel to the process of gathering information on user needs and starting from the premise and initial agreement that a conversational service or bot is a very appropriate technological solution for the context of the problem, the aim is to analyse the market and decide which could be the most suitable development platform for this type of service, based on the requirements detected.

In this sense, a study is carried out on different platforms from the technological part of the multidisciplinary team developing the project, reaching the conclusion that IBM Watson, IBM's chatbot development platform [32-34], presents a series of advantages that differentiate it and make it the most suitable for our needs, compared to other alternatives, such as Amazon's Alexa [35] or Apple's Siri [36]. All of them offer question and answer assistance functionality, but the two mentioned are limited to use on iOS or Amazon devices, respectively, while Watson can implement solutions for any mobile platform. In addition, these platforms are voice-only, and in order to make them as accessible as possible, Watson allows for both voice and text interaction, with conversion between channels, and even images if needed.

Figure 1 shows schematically, the modular organisation of this platform, which allows it, by means of conversion tools, to interact indistinctly through different channels.

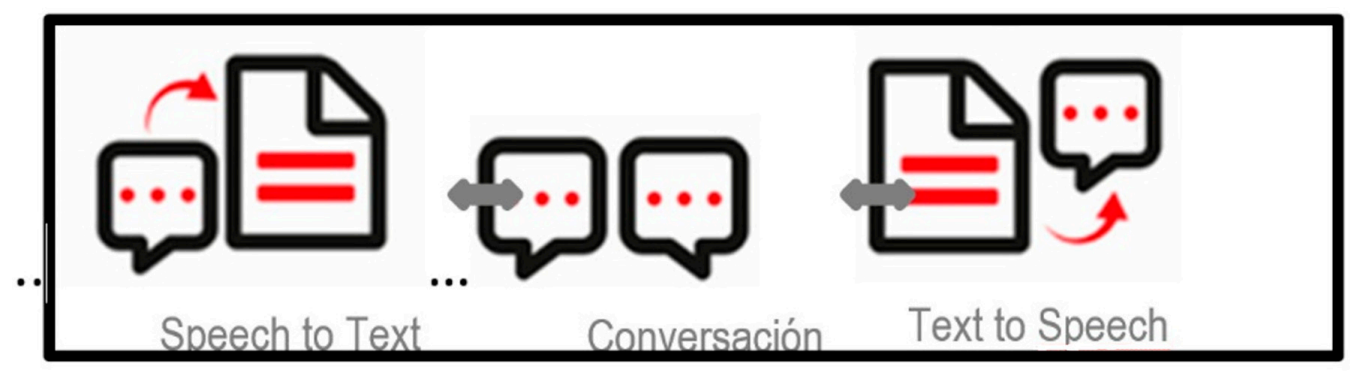

Figure 1. IBM Watson interaction. 
Finally, another great advantage of Watson is that it is defined as an artificial intelligence platform. In practice, this allows for a more natural conversation by using, among other things, its own databases fed from different sources, such as encyclopaedias, news or dictionaries. All these features can also be used to enrich the interaction with the user.

Having concluded from the analysis of development platforms that the best alternative is IBM's Watson, the design of the solution already takes into account the characteristics of this particular platform.

\subsection{Design of the Conversational Logic of the Tool}

The basis of the implementation of a conversational bot is the definition of the conversational logic or thread that supports it. Therefore, the specification of this logic becomes a key task of the development methodology. This logic will be deduced primarily from the analysis of the information and specification obtained on how to work on the development of social skills in the first stage of the interviews.

Although the conversational logic is deduced fundamentally from the information gathered, in practice this logic subsequently must be adapted to the development tool itself, to Watson in our case. In other words, the logic must be translated into the conversational services offered by Watson, mainly using the Watson Assistant modules for the creation of the conversational assistant and implementation of dialogues, as well as the Text to Speech and Speech to Text modules, referred to above, for interaction through different channels.

Specifically, to design the conversational logic in Watson, some key concepts defined in the tool must be taken into account, such as Intentions (intents) to interpret what the user has wanted to say; Entities or user inputs (inputs) formed by the words, categories or phrases that determine the chatbot's response; Dialogues or structure of the conversation itself, i.e., what we want the chatbot to say when it identifies the intention or what the user has wanted to say.

In short, it is a matter of translating or adapting the requirements of the application to this specific nomenclature of the tool, and this will be the main task of the next stage of development, but before and as a result of this third stage, we will obtain the tree that defines the conversational logic that must be translated into the terms of the tool itself.

\subsection{Tool Development and Implementation}

As just mentioned, the implementation of the tool in the end is mainly based on transforming the requirements specification and conversational logic in the form of a tree obtained in the previous stage, into the specific language or terms of the tool.

In this sense, the main task for the development of the tool consists in the implementation of the chatbot Dialogue. This implementation in turn will be based on the definition of the relevant Entities or inputs by the user, in voice or text format, the Intentions that are deduced from them, and combining all of this through the Dialogues, to give the most appropriate response.

By implementing all this in the tool, the result of this phase will be a first chat bot prototype.

\subsection{Training of the Chatbot, Tests with Users and Feedback to Improve the Tool}

Once the first functional prototype has been implemented and obtained, the aim is for the users themselves to test it and help to enrich and improve it with their feedback, until a final product is obtained and is approved by users.

To this end, a script has been designed and different levels of testing have been carried out. The first, alpha tests, where users test the tool with the research team in front of them, who note down the deficiencies and improvements they suggest based on its use. The second, beta testing, where users test the application independently and report their comments. On this basis, the tool has been refined until the current version is obtained. 


\subsection{Use of the Chatbot in the Real Environment}

The last stage is aimed at disseminating the tool for its exploitation and use by end users.

Although it is true that a testing phase has been carried out that has allowed the tool to be refined to its current version, it is also true that it would have been desirable to extend the testing process to more users. In the end, this proved impossible due to the limitation imposed by the pandemic itself on access to end users. For this reason, what is now considered to be the final version is probably subject to further improvement when conditions permit it and more users can continue to test the tool.

The tool is therefore already in use by some Insolamis users, and other entities have shown interest in it, but it has not yet been uploaded to public access channels.

\section{Results}

The main result obtained is an Android mobile application that integrates intelligent conversational services, whose interaction can be done by voice or text, adapted to the diversity of users and responds to the need raised by people with intellectual disabilities to develop and improve social skills. At the same time, it turns real users into active agents of change, facilitating the provision and creation of accessible resources.

As shown in Figure 2, which shows the main interface of the application, it is a simple and user-friendly, with a textbox and buttons to enter text and/or voice, displaying on screen the conversation held as a chat.

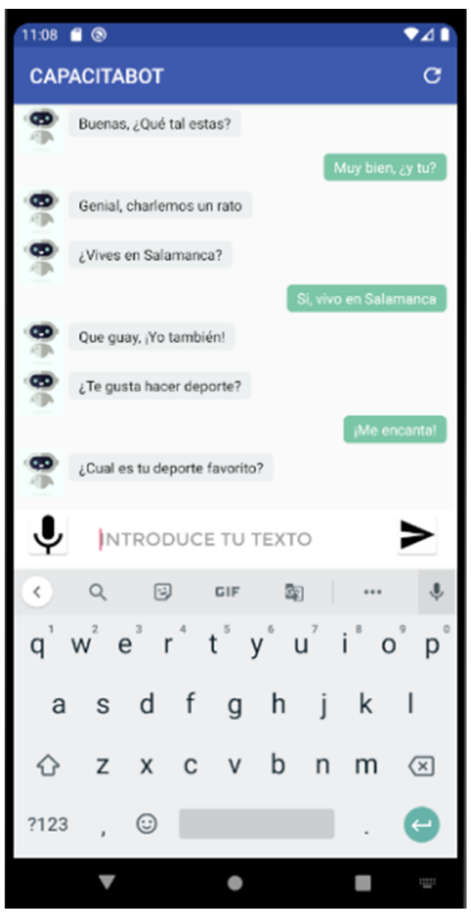

Figure 2. Screenshot of CapacitaBOT Main interface.

The first results obtained after carrying out the alpha and beta tests by the users seem promising, although a broader study and for a longer period of time with more users could allow us not only to continue improving the tool, but also to specify more precisely to what extent the social skills of people with disabilities improve with the use of the tool.

Although the application itself is the main result, it is achieved through intermediate sub-results that can also be considered as important contributions of the tool. These sub-results are detailed below. 


\subsection{Architecture for a Tool That Integrates a Conversational Bot}

An architecture has been proposed (Figure 1), in which conversational services, such as Watson Assistant, are integrated with text-to-speech and speech-to-text services. These are integrated into a mobile application, but they could be incorporated into a web or any other support in the same way.

The proposed system can be extrapolated for the development and implementation of conversational bots for use in other educational contexts for people with disabilities, because it facilitates communication and offers different formats of use.

\subsection{Conversational Requirements and Conversational Logic}

Based on the observation protocols carried out with the target users of the tool, the specific conversational requirements for the target users of the tool have been identified. Figure 3 shows an example of these requirements. Once the requirements have been identified, the conversational logic to be implemented is defined. Figure 3 shows an example of a conversational flow for a simple conversation to work with the target users. This diagram shows how, first of all, an initial greeting is made to the user. Once the user answers and once this initial greeting phase is over, different conversation topics would be raised, always depending on the previous answers given. For this example, topics such as sports, food or family have been chosen. When the user has conversed with the tool on one topic, he/she would move on to another, and so on, until the end of the conversation with a farewell or final message. The users' interest in following the conversation has been one of the key aspects to continue using the application.

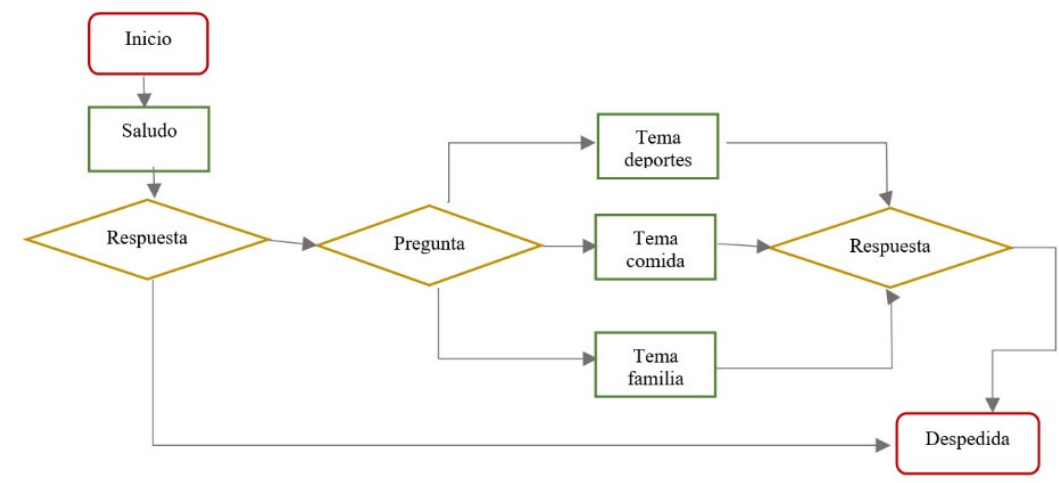

Figure 3. Example of conversational logic for a basic conversation.

Both the conversational requirements and the conversational logic or flow represent a further result of the present work. They could be used and implemented in any other existing or future technology, such as Alexa or Siri.

\subsection{Dialogs}

For each type of conversation, or requirement and conversational logic, all the necessary dialogues have been defined in the Watson tool.

In relation to the dialogues, which use not only the intents but also the context of the application to give an answer, all the entities have been identified and implemented, or in other words, the possible input information relevant to the user's target. Figure 4 shows some examples of entities defined in the implemented chatbot. For example, the @Feelings (@sentimientos) entity identifies all possible responses (happy-contento, sadtriste) to a conversation about feelings, helping to direct or guide the conversation in one flow or another. 


\begin{tabular}{|c|c|c|}
\hline$\square$ & @respuestasLogicas & Mal, No se, Genial, Muy bien, Bien, Regular, Fatal \\
\hline$\square$ & @respuestasSiNo & No, Si \\
\hline$\square$ & @Sentimientos & Contento, triste \\
\hline
\end{tabular}

Figure 4. Example entities implemented in Watson Assistant.

In Figure 5 we can see an example with a piece of dialog. In it, we can observe that when the user answers "SI" (yes) to the question " $¿$ Te gusta hacer deporte? (Do you like to do sports?), the flow goes to DeporteFavorito (FavouriteSport) option. However, if the answer is another different to "SI" (yes), the conversation will be guide to a different way. After DeporteFavorito, another question is done in a similar way, guiding the dialog to a one or other flow, always taking into consideration the answer of the user. This schema will be repeated until the dialog is finished.

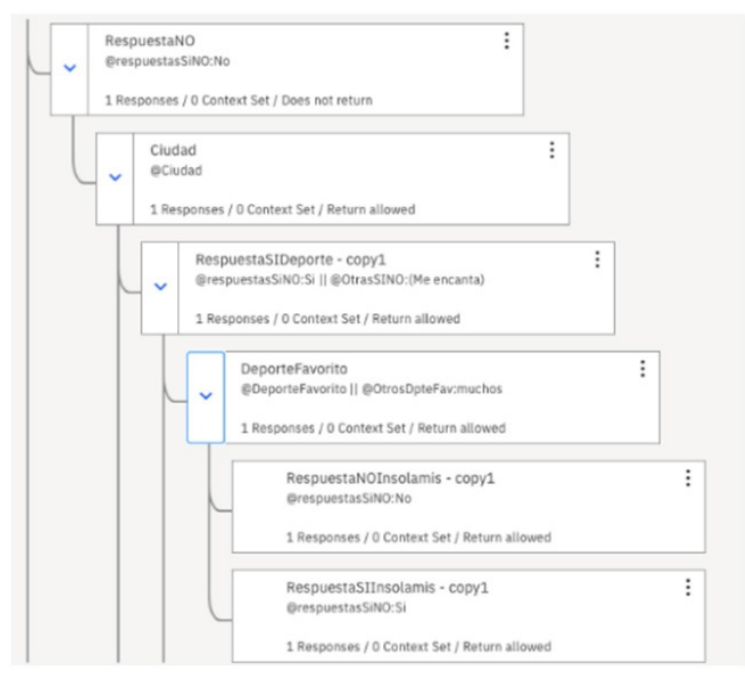

Figure 5. Example of a Dialogue in Watson Assistant.

Figure 5 shows as an example a part of a dialogue. In it, when answering YES to the question Do you like doing sport? (¿Te gusta hacer deporte?), the user accesses FavouriteSport (DeporteFavorito), and the conversation follows a different path in another case. From FavouriteSport (DeporteFavorito) another question is asked in a similar way, the dialogue taking one path or another depending on the answer. This scheme is repeated until the end of the dialogue.

Figure 6 shows possible responses "Cool!", “Great!", “That's very good!" ("iQue guay!", “;Genial!", “Eso está muy bien!") to the @FavouriteSport (@DeporteFavorito) entity recognised by the assistant. In this case, they have been set up as random answers, so the assistant will choose an answer randomly. As the language is simple and easy to read, the users have followed the route through the different topics with great motivation.

The dialogues themselves are a result, as they can be integrated into any other medium/platform such as a desktop application, the web, etc. 


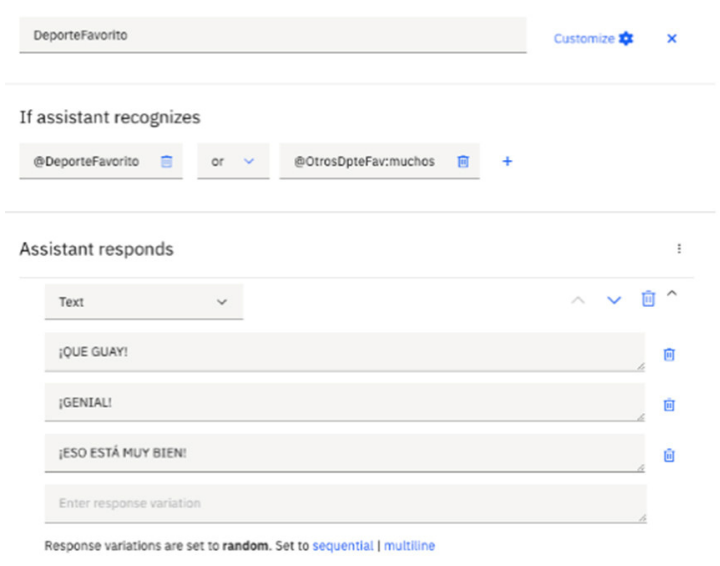

Figure 6. Example of responses to a user intervention in Watson Assistant.

\section{Discussion}

The World Health Organisation (WHO) declared a COVID-19 pandemic, due to the spread of the SARS-CoV-2 outbreak, which led to a health crisis with repercussions on education and social relations [37].

During the period of confinement, educational institutions and associations were closed, leaving persons with disabilities with communication barriers and limitations to cognitive accessibility, as well as lost opportunities to develop social skills outside the familiar environment.

Faced with this situation, the design and implementation of a conversational bot (CapacitaBOT) becomes an aid that allows, through its features, to improve the communication skills of people with intellectual disabilities. The conversational bot responds to the initially proposed objectives by favoring educational and social inclusion through the acquisition of communication skills, while at the same time improving the quality of life of these people.

In this way, it can be considered as an educational tool that allows the integration of innovation, inclusion and quality in education for people with intellectual disabilities. The tool empowers these people for real life situations, making it also a resource that allows the application of active methodologies as it facilitates the learning of social skills. Finally, all the contributions of the tool are in line with the objectives of sustainable development, as it is a tool that facilitates accessibility for people with disabilities, who more than ever have been affected by the social isolation caused by the COVID-19 crisis.

The previous work with potential users has allowed us to know the needs of people with intellectual disabilities.

The choice of IBM's chatbot development platform also demonstrates the advantages obtained, on the one hand, by allowing communication through different channels (voice and text), as well as a more fluid and natural conversation, thanks to its artificial intelligence features, all of which is fundamental to the needs of the tool's target group.

Finally, it is worth mentioning that the basic functionalities and features of the intelligent chatbot can be extrapolated to work on social skills with people who have learning, communication or social interaction difficulties. In short, the technological solution is therefore very appropriate for the problem context that was initially identified and has the advantage of being able to adapt to different educational contexts and realities.

\section{Conclusiones}

Ban Ki-moon [38] pointed out that technology could help people with disabilities to use their full potential in their communities and workplaces, and thus make the most of their abilities.

Seven years on, progress has been made and technologies such as Artificial Intelligence have made great strides, so much so that it is emerging as a key tool for inclusion in a society that tends to push people with disabilities to the margins. 
As we have seen in the literature, there is currently a lack of tools that allow people with intellectual disabilities to develop and improve their social skills [9]. In order to alleviate this problem, a tool has been developed and presented in this paper, CapacitaBOT. It allows people with intellectual disabilities to develop and enhance their social skills and to develop with greater autonomy through the training of communication skills. It is an intelligent tool that aims to help people with intellectual disabilities to initiate, maintain and end an interaction with others, to understand and respond in different situations and contexts and to be able to respond to the demands of others. In short, to know, control and improve the social skills of people with intellectual disabilities using technology and specifically artificial intelligence service platforms as a tool. In short, the users who benefit from this application can acquire a better understanding of the context so that they can act on it, favoring interpersonal relationships.

The main difficulty encountered has been the time limitation caused by the health emergency to carry out a more exhaustive follow-up of each of the selected users, thus reducing the conversational contexts collected by the application. Likewise, the time limitation has prevented the tool from being adequately trained to "learn" from the users themselves, generating new questions and answers automatically, which would have greatly enriched the tool. However, this drawback has been solved with the collection of previous information, with observation protocols and semi-structured interviews which have served to be designed and implemented on the Watson platform and integrated into a mobile application conversational flow with different pre-questions and multiple answers to each one, simulating a conversation as real and natural as possible.

Once the functionality of the application has been tested and its strengths and weaknesses have been identified, it has been considered very useful to include future actions that will enable the generalisation of the use of this mobile tool to a wide range of contexts. In the same way, and with the aim of reaching the maximum number of users, it is desirable to develop it on other systems or platforms such as iOS devices or for the Web.

The ultimate goal of the project is the integration of people with intellectual disabilities and this tool is the means that facilitates the development of their social skills.

Author Contributions: Conceptualization, M.M.-S., A.C.M., L.S.B. and A.M.F.G.; methodology, M.M.-S. and A.M.F.G.; software, M.M.-S. and A.M.F.G. validation, A.C.M. and L.S.B.; formal analysis, M.M.-S. and A.M.F.G.; investigation, M.M.-S., A.C.M., L.S.B. and A.M.F.G.; writing-original draft preparation, M.M.-S., A.C.M., L.S.B. and A.M.F.G.; writing-review and editing, M.M.-S. and A.M.F.G.; supervision, A.C.M. and L.S.B.; project administration, A.M.F.G. All authors have read and agreed to the published version of the manuscript.

Funding: This research received no external funding.

Institutional Review Board Statement: Not applicable.

Informed Consent Statement: Informed consent was obtained from all subjects involved in the study.

Conflicts of Interest: The authors declare no conflict of interest.

\section{References}

1. AAIDD. Discapacidad Intelectual: Definición, Clasificación y Sistemas de Apoyo; Alianza Editorial: Madrid, Spain, 2011.

2. Organización Mundial de la Salud. Clasificación Internacional del Funcionamiento, de la Discapacidad y de la Salud (CIF); Versión Abreviada; IMSERSO: Madrid, Spain, 2018.

3. Heredero, E.S.; y Carralero, A.O. Experiencias y recursos con las TICs para la atención al alumnado con necesidades educativas especiales. Acta Sci. Educ. 2014, 36, 279-286. [CrossRef]

4. Cabero, J.; Córdoba, M.; y Fernández, J. Las TIC Para La Igualdad; Publidisa: Sevilla, Spain, 2007.

5. Solla Salvador, C. Guía de Buenas Prácticas en Educación Inclusiva; Save de Children: Madrid, Spain, 2013.

6. UNESCO. Informe de Seguimiento de la Educación en el Mundo 2020 Inclusión y Educación: Todos, sin Excepción; UNESCO: Paris, France, 2021.

7. Organización de Naciones Unidas. Convención Internacional Sobre los Derechos de las Personas con Discapacidad. CERMI. 2006. Available online: https:/ / bit.ly/3ssTELv (accessed on 12 June 2020). 
8. Jefatura de Estado. Instrumento de Ratificación de la Convención Sobre los Derechos de las Personas con Discapacidad, Hecho en Nueva York el 13 de Diciembre de. Boletín Oficial del Estado (BOE), 96, Spain, 21-4-2008. 2006. Available online: https:/ / bit.ly /3cq0onO (accessed on 30 September 2020).

9. Berrios, O. Tecnologías para Personas con Discapacidad Intelectual; Plena Inclusión España: Madrid, Spain, 2020.

10. Geer, D. Malicious bots threaten network security. IEEE Comput. 2005, 38, 18-20. [CrossRef]

11. Yang, Y.; Vlajic, N.; y Nguyen, U.T. Next Generation of Impersonator Bots: Mimicking Human Browsing on Previously Unvisited Sites. In Proceedings of the IEEE 2nd International Conference on Cyber Security and Cloud Computing, New York, NY, USA, 3-5 November 2015; pp. 356-361.

12. Túñez-López, J.M.; Toural-Bran, C.; Cacheiro-Requeijo, S. Uso de bots y algoritmos para automatizar la redacción de noticias: Percepción y actitudes de los periodistas en España. Prof. Inf. 2018, 27, 750-758. [CrossRef]

13. Bonales, G.; Pradilla, N.; y Citlali, E. Chatbot como herramienta comunicativa durante la crisis sanitaria de la COVID-19 en España. ComHumanitas Rev. Cient. Comun. 2020, 11, 1-22.

14. Abdul-Kader, S.A.; Woods, J.C. Survey on Chatbot Design Techniques in Speech Conversation Systems. Int. J. Adv. Comput. Sci. Appl. 2015, 6, 72-80.

15. Wiezenbaum. ELIZA-A computer program for the study of natural languaje communicacion between man and machine. Comun ACM 1966, 9, 36-45. [CrossRef]

16. Wallace, R.S. The Anatomy of A.L.I.C.E. In Parsing the Turing Test; Springer Science+Business Media: London, UK, 2009; pp. 181-210.

17. Khan, S.; y Raza Rabbani, M. Chatbot as Islamic Finance Expert (CaIFE): When Finance Meets Artificial Intelligence. In ISCSIC 2020, Proceedings of the 2020 4th International Symposium on Computer Science and Intelligent Control, Newcastle upon Tyne, UK, 17-19 November 2020; ACM: New York, NY, USA, 2020; Volume 37, pp. 1-5. [CrossRef]

18. Um, T.; Kim, T.; Chung, N. How does an Intelligence Chatbot A_ect Customers Compared with Self-Service Technology for Sustainable Services? Sustainability 2020, 12, 5119. [CrossRef]

19. Hwang, S.; Kim, J. Toward a Chatbot for Financial Sustainability. Sustainability 2021, 13, 3173. [CrossRef]

20. Carisi, M.; Albarelli, A.; y Luccio, F. Design and implementation of an airport chatbot. In GoodTechs '19, Proceedings of the 5th EAI International Conference on Smart Objects and Technologies for Social Good, Valencia, Spain, 25-27 September 2019; ACM: New York, NY, USA, 2019; pp. 49-54. [CrossRef]

21. Perdana, F.; Meidia H y Gunawan, D. Designing Intelligent Personalized Chatbot for Hotel Services. In ACAI 2019, Proceedings of the 2019 2nd International Conference on Algorithms, Computing and Artificial Intelligence, Sanya, China, 20-22 December 2019; ACM: New York, NY, USA, 2019; pp. 468-472. [CrossRef]

22. Frommert, C.; Häfner, A.; Friedrich, J. Using Chatbots to assist communication in collaborative networks. In Collaborative Networks of Cognitive Systems; Springer: Cham, Switzerland, 2018; pp. 257-265.

23. Narain, J.; Quach, T.; Davey, M.; Won Park, H.; y Breazeal, C. Promoting Wellbeing with Sunny, a Chatbot that Facilitates Positive Messages within Social Groups. In CHI EA '20, Proceedings of the Extended Abstracts of the 2020 CHI Conference on Human Factors in Computing Systems, Honolulu, HI, USA, 25-30 April 2020; ACM: New York, NY, USA, 2020; pp. 1-8. [CrossRef]

24. Avila, J.F.; Olano, E.; Minué, C. Dejal@Bot: Un chatbot aplicable en el tratamiento de la deshabituación tabáquica. Rev. Investig. Educ. Cienc. Salud 2020, 5, 33-41.

25. Avila, J.F.; Olano, E.; Minué, C. Nuevas herramientas de comunicación digitales entre profesionales de la salud y pacientes. A propósito del proyecto Dejal@Bot. Rev. Comun. Salud 2019, 9, 55-70.

26. Garcia, G.; Fuentes, M.; Molas, N. Briefing Paper: Los Chatbots en Educación; UOC Repositorio Institucional: Barcelona, Spain, 2018.

27. Georgescu, A. Chatbots for education-Trends, benefits and challenges. Actas eLearning Softw. Educ. 2018, 14, 195-200.

28. Fulmer, R.; Joerin, A.; Gentile, B. Using Psychological Artificial Intelligence (Tess) to Relieve Symptoms of Depression and Anxiety: Randomized Controlled Trial. JMIR Ment. Health 2018, 5, e64. Available online: https://mental.jmir.org/ (accessed on 1 November 2021). [CrossRef] [PubMed]

29. Stiefel, S. The Chatbot Will See You Now: Protecting Mental Health Confidentiality in Software Applications. Colum. Sci. Technol. Law Rev. 2019, 20, 333. [CrossRef]

30. Darcy, A.; Daniel, J.; Salinger, D. Evidence of Human-Level Bonds Established with a Digital Conversational Agent: Crosssectional, Retrospective Observational Study. JMIR Form. Res. 2021, 5, e27868. [CrossRef] [PubMed]

31. Organización de Naciones Unidas. Objetivos de Desarrollo Sostenible. Agenda 2030. ONU, 2015. Available online: https: / / bit.ly/3rsASmj (accessed on 2 January 2020).

32. IBM. Watson Assistant. Getting Started with Watson Assistant. 2020. Available online: https://ibm.co/31CxKdf (accessed on 3 April 2020).

33. IBM Speech to Text. Iniciación a Speech to Text. 2020. Available online: https://ibm.co/3fl7SdY (accessed on 10 April 2020).

34. IBM. Text to Speech Getting Started with Text to Speech. 2020. Available online: https://ibm.co/3w5fGpV (accessed on 3 April 2020).

35. Amazon. Build Skills with the Alexa Skills Kit. 2020. Available online: https://developer.amazon.com/es-ES/alexa (accessed on 10 September 2020).

36. Apple. Siri for Developers. 2020. Available online: https://developer.apple.com/siri/ (accessed on 20 March 2020). 
37. Adhanom Ghebreyesus, T. Alocución de Apertura del Director General de la OMS en la Rueda de Prensa Sobre la COVID-19 Celebrada el 11 de Marzo de 2020. OMS. Available online: https:/ / bityl.co/45Vo (accessed on 15 April 2020).

38. Ki-Moon, B. ¿Puede la Inteligencia Artificial Facilitar la vida a las Personas con Discapacidad? 2014. Available online: https: //www.mapfre.com/actualidad/innovacion/ia-personas-discapacidad/ (accessed on 2 December 2021). 